\title{
Virus-like particles as drug delivery vectors
}

\author{
Michał Zdanowicz ${ }^{1}$ and Jadwiga Chroboczek ${ }^{1,2 \bowtie}$ \\ IInstitute of Biochemistry and Biophysics, Polish Academy of Sciences, Warsaw, Poland; 2 Therex, TIMC-IMAG, CNRS UMR 5525, UJF, Domaine de \\ la Merci, 38700 La Tronche, France
}

\begin{abstract}
Virus-like particles (VLPs) assemble spontaneously during the viral cycle or in heterologous systems during expression of viral structural protein. Depending on the complexity of the VLPs, they can be obtained by expression in prokaryotic or eukaryotic expression system from the suitable recombinant vectors, or formed in cell-free conditions. Moreover, they can be built from proteins of a single virus, or can present the proteins or peptides derived from a virus or cell on a platform derived from any other single virus, thus forming chimeric VLPs. VLPs are best known for their immunogenic properties, but the versatility of VLPs allows a wide variety of applications. They are lately in the centre of investigations in vaccinology, drug delivery and gene therapy. This review focuses on utilization of VLPs for drug delivery.
\end{abstract}

Key words: VLPs, vaccines, virus

Received: 17 February, 2016; revised: 12 May, 2016; accepted: 15 May, 2016; available on-line: 30 July, 2016

\section{INTRODUCTION}

VLPs are best known for their immunogenic properties, but the versatility of VLPs allows a wide variety of applications. They are lately in the centre of investigations in vaccinology, drug delivery and gene therapy. This review focuses on utilization of VLPs for drug delivery.

The term "VLP" has been in use for at least 40 years, defining various biological objects. This includes empty structures of viral origin, infectious or non-infectious viruses, interfering viral particles with some structure modifications and non-infectious, self-assembled gene products acquired from the expression of viral structural genes in heterologous systems. In this review the term VLP pertains to self-assembled structures, produced during expression of viral structural proteins.

Virus-like particles (VLP) are multimeric nanostructures, which means that the particle size is between 0.1 and $100 \mathrm{~nm}$ in each spatial dimension. These, sometimes multiprotein, particles are constructed from viral structural proteins, capsomers, and are free of any genetic material. Capsomers may be bound to each other by covalent interactions such as disulphide bonds or electron sharing, or non-covalent bonds such as hydrogen bonds, ionic interactions, Van der Waals forces. Some of VLPs are formed in a calcium-dependent manner, whereby the disulphide bonds increase stability of calcium binding (Ishizu et al., 2001). Abundance of those ions strengthens the structure of the particle, while removal of them loosens the interactions between capsomers resulting in decreased particle stability or its disintegration to capsomers. The VLPs display remarkable cell entry pro- pensity, which stems from the fact that they are formed from functional viral proteins that are responsible for cell penetration by the virus.

Over 100 VLPs originating from microbial, plant, insect and mammalian viruses have been produced and characterized since the beginning of the 1980s, derived from 35 different virus families (Zeltins, 2013). Like naturally occurring viruses, VLPs can be enveloped or non-enveloped, spherical or filamentous (Fig. 1). VLPs assemble spontaneously during the viral cycle or in heterologous systems during expression of viral structural protein. Depending on the complexity of the VLPs, they can be obtained by expression in prokaryotic or eukaryotic expression system from the suitable recombinant vectors, or formed in cell-free conditions. Moreover, they can be built from proteins of a single virus, or can present the proteins or peptides derived from a virus or cell on a platform derived from any other single virus, thus forming chimeric VLPs.

The efficiency of VLPs expression is quite high. Expression yield in eukaryotic systems can be similar to that obtained in a bacterial systems. Because of high molecular weight of VLPs, they can be easily purified from extracts of the expressing cells by sucrose density centrifugation or size-exclusion chromatography, often with additional separation steps to remove unnecessary cellular components (Chroboczek et al., 2014).

\section{VLP VACCINES}

The most common use of VLPs is as a platform to present epitopes to the immune system. In majority of cases this is achieved by modification of the VLP gene sequence in such a way that during expression the capsomeres with embedded foreign epitope will form VLPs. Larger protein domains might be difficult to present on VLPs as display of large proteins may disrupt VLPs folding process. In such situation, the chemical conjugation can be the solution (Spohn et al., 2010).

Among the advantages of VLP vaccines there is the fact that they can be successfully produced in various expression systems. In many cases such VLPs have structural characteristics and antigenicity similar to the parental virus and are quite often amenable to insertion of endogenous or foreign antigenic sequences, allowing production of chimeric VLPs exposing the antigen on

e-mail: jadwiga.chroboczek@imag.fr

Abbreviations: VLP, virus-like particle; HBV, hepatitis B virus; HPV, human papilloma virus; MBP, maltose-binding protein; HCC, hepatocellular carcinoma; RTA, ricin toxin A-chain; TK, thymidine kinase; GFP, green fluorescent protein; BLM, bleomycin; Ad, adenovirus; $\mathrm{Dd}$, dodecahedron; Dox, doxorubicin; cap, cap $\left(\mathrm{m}^{7} \mathrm{G}\right)$ structure analogue 


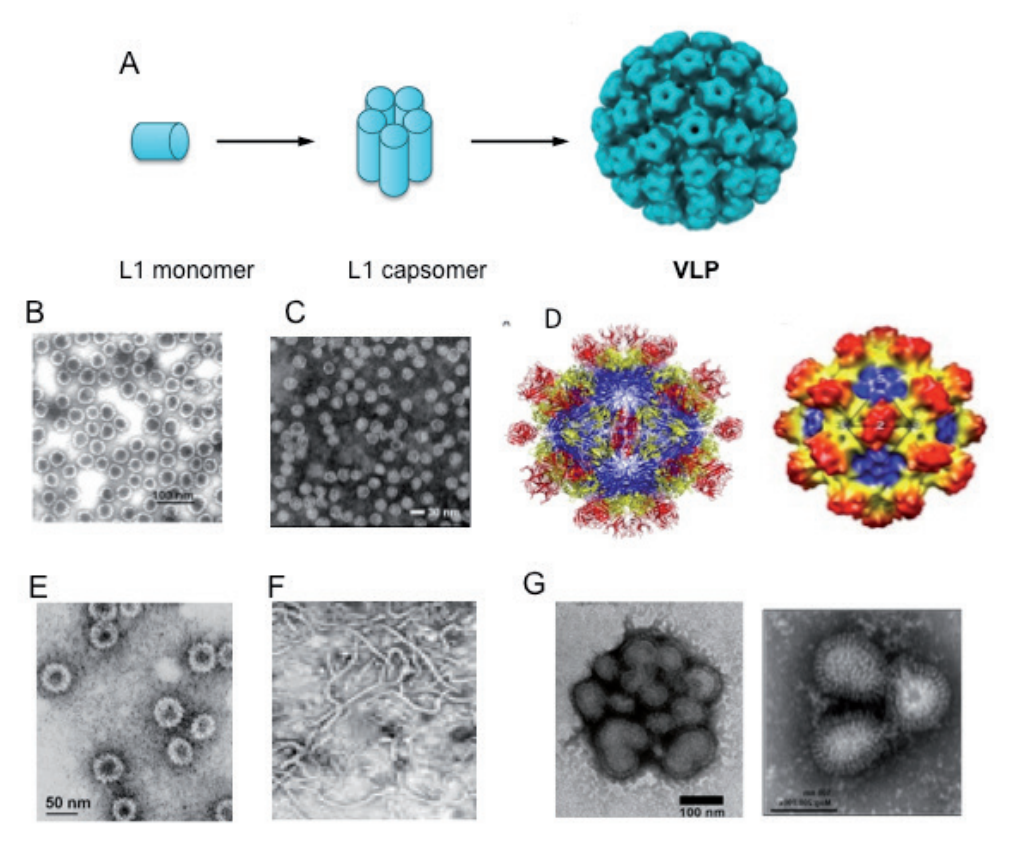

Figure 1. Virus-like particles.

(A) Diagram of assembly of HPV VLP from the L1 capsid protein. Five L1 monomers form spontaneously one pentameric L1 capsomer, and 72 of such capsomers selfassemble into a VLP. Adapted from German Cancer Research site http://www.dkfz. de. (B) Electron micrograph of the VLPs built of ssRNA bacteriophage AP205 coat protein expressed in E. coli. These VLPS serve as the platform of a conjugate vaccine against West Nile virus (WNV) consisting of recombinantly expressed domain III (DIII) of the WNV E glycoprotein chemically crosslinked to the AP205 VLP. After Spohn et al., 2010. (C) Electron microscopy of the VLPs built of the coat protein of phage $\mathrm{Q} \beta$ expressed in E. coli. Source: http://www.cytos.com/vlp-platform/. (D) Structure of the hepatitis E virus-like particle (HEV VLP). Left panel shows the atomic structure of HEV VLP. The 3 domains, S, P1, and P2 are coloured blue, yellow, and red, respectively. Right panel - cryo-EM reconstruction at $14 \AA$ resolution. After Guu et al. (2009). (E) Transmission electron micrographs of polyomavirus-like particles (PyVLPs) produced in insect cells and then purified by sucrose density sedimentation. After Shin and Folk, 2003. (F) Ebola VLP visualized by EM. Reproduced from somapps. med.upenn.edu site. (G) Influenza enveloped VLPs. Left panel - EM of negatively stained A/Anhui/1/2013 (H7N9) VLP, assembled from HA and NA proteins expressed from separate baculovirus vectors in insect cells. After Smith et al., 2013. Right panel - influenza VLPs produced in a baculovirus system with the sequences of the genes for HA, NA, and M1 of the 1918 pandemic virus. After Perrone et al., 2009.

their surface. VLPs provide the spatial structure similar to the parental virus native structure, thereby enhancing the production of neutralizing antibodies. (Frietze et al., 2014).

Some VLP vaccines have been already licensed and commercialized. The prophylactic human vaccines against hepatitis $\mathrm{B}$ virus (HBV) and human papilloma virus (HPV), both based on VLPs derived from these viruses have been FDA-approved and are in use. Additionally, an anti-malaria vaccine consisting of hepatitis B VLP presenting multiple copies of a secreted protein (circumsporozoite protein, CSP) of the Plasmodium falciparum malaria parasite should soon be approved. Other VLP vaccines targeting influenza virus, rotavirus or human immunodeficiency virus are undergoing preclinical development (Kushnir et al., 2012).

\section{WHY VLPS ARE GOOD DRUG DELIVERY VECTORS?}

Besides carrying peptides/proteins or other active molecules exposed on the outside, VLPs possess the ability to encapsulate or attach proteins, nucleic acids or other small molecules. They can thus act as delivery vessels to target specific cells, tissues or organs, liberating the embedded, attached or encapsulated elements inside the cells (Lua et al., 2014). For entry into the cell VLPs employ the energy-using receptor-mediated endocytosis. During endocytosis cell engulfs molecules from the plasma membrane together with the extracellular fluid in intracellular membrane-bound vesicles that subsequently traffic through the cell. Intracellular release of vesicles requires the scission by the dynamin GTPase. Next, liberated vesicles travel along cytoskeleton to fuse with early endosomes. Eventually, endosomal vesicles detach from the early endosome to form multivesicular bodies, which then mature into late endosome. Finally, late endosomes fuse with prelysosomal vesicles containing acid hydrolases, creating lysosomes, in which degradation of the majority of cargo would occur (Bareford et al., 2007). Molecules internalized from the plasma membrane together with synthetic nanoparticles, would follow this pathway all the way to lysosomes for degradation. However, the lysosomal degradation precludes proper drug delivery (Shete et al., 2014). Indeed, success in intracellular delivery of active agents remains limited. The Achilles heel of many modern therapies is drug delivery; about $40 \%$ of newly developed drugs are rejected because of poor bioavailability. Delivery vectors can be built using approaches based on macromolecular chemistry and physics. These chemical vectors theoretically fulfil all the requirements necessary for delivery targeted to specific diseased cells in vivo. However, the numerous limitations of nanobioconjugates include toxicity, inability to deliver enough molecules to the cytoplasm and lack of biodegradability (Sebestik et al., 2011; Jian et al., 2012; van der Berg \& Dowdy, 2011). Contrary to that, VLPs, like original viruses, have the ability to escape the endosome before lysosomal degradation occurs (Fender et al., 1997), the feature that is unique to viruses and VLPs. Another argument in favour of VLPs use is their lack of original viral genome; there is no risk associated with virus replication or formation of viable virus through re-assortment. Because of that, safety problems connected with whole-virus administration, like reversion of attenuated virus to infectious one or virus escape from production facility, are alleviated (Crisci et al., 2012).

Various active molecules can be encapsulated inside VLPs. The inner surface of the particle can be modified with chemical agents so that molecule to be transported will bind to internal VLP surface during the assembly process (Ma et al., 2012). For this, we need to know some features of VLP structure, namely what is the chemical nature of bonds holding VLP together. For example, cowpea chloric mottle capsid can be subjected to a $\mathrm{pH}$ and metal ion-dependent reversible structural transition. At the $\mathrm{pH}>6.5$ (swollen state) the inside of the VLP is uncovered and thanks to channels (pores) between the capsomeres, cargo molecules can enter freely. At $\mathrm{pH}<6.5$ pores of the capsid are closed (contracted state) and release of cargo molecules in vitro is thwarted. This method of molecular entrapment was 
used to entrap anionic polymers (Douglas et al., 1998). As the relative efficacy of many drug combinations is ratio-dependent, it would be important to control the dose of encapsulated drug. However, no data are available in the literature indicating that some dose controlling can be exerted during drug encapsulation by VLPs.

Beside trapping molecules inside the VLP, ligands can also be attached to the VLPs surface. Structure of viruses used in VLP development is often well known. This allows for localization of $\mathrm{N}$ - and C-termini of their subunits and identification of external loops able to uphold genetic insertions. Then protein fusion can be used to introduce heterologous peptides/proteins on the surface of VLP. Molecules can also be linked to the exterior of the VLP by chemical modification of the functional groups contained in the structural proteins forming the capsid. This strategy allows to functionalize surface of the VLPs, granting them new specificity, tropism or physical properties. For example, carboxylate groups on the outer surface of cowpea mosaic virus were used for binding carboxylate-selective chemical dye which allowed the detection of applied VLPs by UV/visible absorption (Grasso \& Santi, 2010).

However, there is a feature of VLPs that might be deleterious to their role as drug delivery vehicle. This is induction of the immune response by proteinaceous particle application, which might significantly limit the use of VLP-based drug delivery. Repetitive, dense and ordered structure of VLPs activates innate immunity by pattern recognition and stimulates a potent humoral immunity; the mammalian immune system is highly attuned to recognizing particles in the size range of viruses (20-300 nm). In addition, the VLPs also tend to be uptaken by dendritic cells and transported to lymph nodes, which is the base for building a T-cell response that plays a central role in cell-mediated immunity. The immune response may hinder the VLP use for drug delivery (Grasso \& Santi, 2010). However, it is important to remember that some proteinaceous agents are currently used in medicine, like insulin in diabetes or asparaginase for the acute leukemia treatment. Despite building up immune response, benefits of using those agents might far more surpass the possible disadvantages. They could be of use upon the failure of classical chemotherapy that targets and kills the drug-sensitive cells, allowing the rise of drug-resistant cells.

In conclusion, VLPs display many features that make them unique drug delivery vectors. The designated drug can be encapsulated inside the VLP or attached to its surface. Peptide-based cargos can be co-expressed in multiple copies together with viral structural proteins and incorporated into the VLP during the self-assembly process. VLPs utilize endocytosis as an efficient way of transporting the drug to the cells and liberation into the cytoplasm. It would be interesting to know if VLPs, similarly as parental viruses are able to spread from cellto cell. As they are slowly removed from the cell thanks to proteolytic mechanisms, they are biocompatible. Although VLPs can induce immune response, significant increase in drug bioavailability may compensate this disadvantage.

\section{DRUG TARGETING}

The goal of targeted drug delivery is to transport a drug directly to a specific tissue or body site. It should also have prolonged interaction with the tissue to maximise the amount of transported drug. Targeted drug de- livery is important for a few reasons. Firstly, delivering a drug directly to a particular cell or tissue can reduce harmful side effects. In addition, direct targeting of a certain site of body might help in dose sparing or result in lower number of applications. Some VLPs display a natural tropism to certain tissue, which derives from the parental virus. For example, as Hepatitis B virus (HBV) is a small virus that infects the liver, HBV VLP can be thus a vector targeting hepatocytes (Shlomai et al., 2009). Similarly, rotavirus displays a marked tropism for the gut (Estes \& Kapikian, 2007), which permitted hypothesis that the rotavirus VLP could be a specific gut-delivery vector (Cortes-Perez et al., 2010). However, in majority of cases VLPs display rather large natural tropism (like an affinity to sialic acids or heparan sulphates, molecules found on many types of cells and tissues) and such tropism should be restrained. The more specific targeting function is usually obtained through attaching receptorrecognizing domains to the drug carriers. As described above for chimeric VLPs, targeting domain can be chemically attached to the surface of VLP by conjugation, or the sequence of the domain can be genetically inserted and then expressed in the exterior termini/loops of the VLP components (Rynda-Apple et al., 2014). Therefore, to obtain targeting vector, we should know the cell receptors for a given VLP, which are usually parental virus receptors. The viral receptors are surface proteins on the target cell that interact with viral envelope proteins of the viruses allowing them to be attached and internalized into the host cell. To detect the receptor, surface antigen of the virus should be linked with easily detectable marker. For example, the surface antigen of hepatitis B virus (HBV) was expressed in form of a fusion with the maltose binding protein (MBP). Binding to cell receptors could be then detected with anti-MBP antibodies using flow cytometry analysis. By this approach, receptors responsible for HBV antigen surface binding and for cellular HBV entry were successfully detected (Park et al., 2000).

\section{EXAMPLES OF DRUG DELIVERY WITH VLPS}

\section{Cell-specific delivery}

Bacteriophage MS2 VLPs, which self-assemble in the presence of nucleic acids, were used to selectively deliver chemotherapeutic drugs, siRNA cocktails, and protein toxins to human hepatocellular carcinoma (HCC). The specific recognition of HCC was achieved thanks to VLP modification with SP94 peptide, which binds to HCC enabling targeting of those particular cells (Ashley et al., 2011). VLPs with doxorubicin, cisplatin, and 5-fluorouracil selectively killed the Hep3B cells, a HCC cell line. VLPs containing siRNA cocktail, which silences expression of cyclin, inhibited growth and promoted apoptosis of Hep3B cells. Lastly, VLPs loaded with ricin toxin Achain (RTA) killed almost every Hep3B cell, while control cells were left intact (op. cit.). Those results show again that VLPs have the ability to encapsulate variety of cargos and can induce cytotoxicity of targeted cells. It is important to note, though, that those studies were not conducted on animal model.

\section{Selective elimination of HIV-1-infected cells}

VLPs formed of vesicular stomatitis virus G glycoprotein carrying the non-pathogenic mutant of a HIV-1 virulence factor Nef, Nef7, in fusion with herpes simplex 
virus-1 thymidine kinase (TK), were able to successfully enter the HIV-1-infected cells. The delivery of VLP with Nef7/TK caused cell death when HIV-infected cells were treated with ganciclovir (which is be converted by TK to active compound). These VLPs were also proved to be effective against non-replicating, HIV-1-infected primary human monocyte-derived macrophages. It seems that HIV-targeting VLPs are a promising candidate for killing the HIV-1-infected cells (Peretti et al., 2006).

\section{Delivery of GFP}

Rotavirus VLPs were produced in a baculovirus expression system and used to deliver the green fluorescent protein (GFP) to the kidney MA104 cells or intestinal cells from healthy mice (Cortes-Perez et al., 2010). As mentioned above, rotavirus displays a marked tropism for the gut. The rotavirus VLPs successfully entered

A
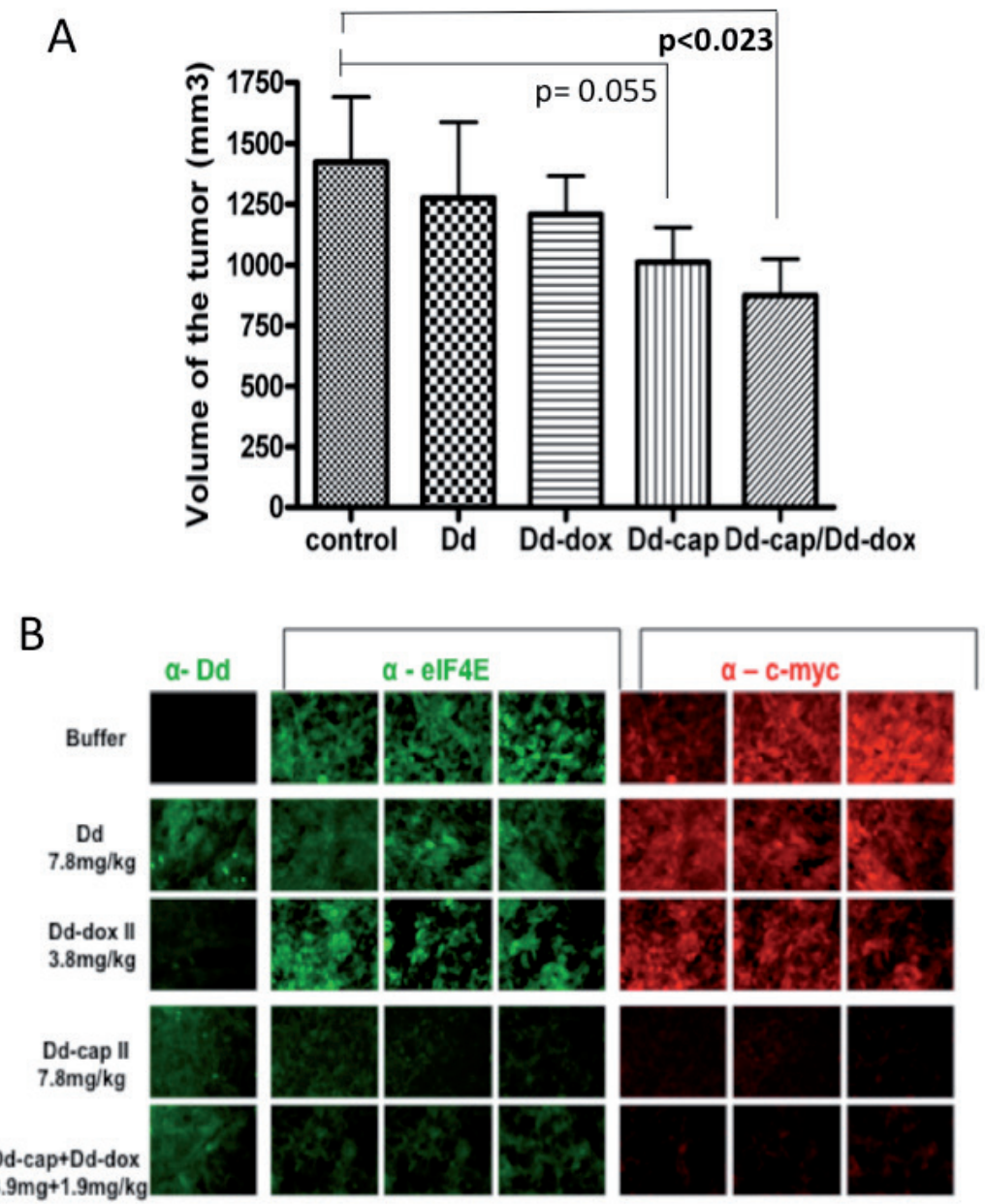

Figure 2. Effect of Dd conjugates in an orthotopic rat model of HCC.

(A) The decrease in tumour growth relative to control untreated animals. The groups of 6-7 animals received Dd (7.8mg/kg body weight), Dd-dox II (3.8 mg/kg), Dd-cap II (7.8 mg/kg), or the combination of Dd-dox II (1.9 mg/kg) with Dd-cap II $(3.9 \mathrm{mg} / \mathrm{kg})$. The control group received buffer $/ 150 \mathrm{mM} \mathrm{NaCl}(6 \mathrm{ml} / \mathrm{kg})$. The agents were administered by intraperitoneal injection once a week for five weeks. Rats were euthanized on day 40 after the first injection. Tumour volume was calculated ex vivo (tumour volume $=(4 / 3)^{*}$ pi*radius $1 *$ radius $2^{*}$ radius 3 ). Tumour growth was compared by applying the Student's $t$-test, which showed that the difference between the control and the rats treated with a combination of Dd-cap and $\mathrm{Dd}$ dox was statistically significant $(p=0.023)$, while the results obtained for the animals treated with Dd-cap were on the border of statistical significance $(p=0.055)$. (B) Immunofluorescence analysis of frozen sections of liver tumour tissue after application of Dd and its conjugates to the animals. The immunohistochemistry was carried out on frozen tissue sections of HCC tumours stained for Dd (first column, in green), elF4E (next 3 columns from different sections, in green) and c-myc (the last three columns, in red). After Zochowska et al. (2015).
MA104 cells and delivered the GFP protein. Furthermore, intragastric administration of fluorescent VLP in les. Those results provide an in vivo evidence of the potential of these nanoparticles as a promising candidate Delivery of bleomycin

Bleomycin (BLM), an anticancer antibiotic causing

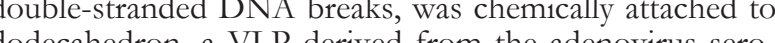
type 3 (Ad3) and formed from Ad3 protein responsible gate induced death of transformed cells by causing DNA ks, similarly as free BLM (Zochowska et al., 2009). Effective cytotoxic concentration of BLM delivered with lower than that of free bleomycin. These results show that use of VLPs for drug delivery have the potential to drastically improve bioavailability of the cargo.

\section{Delivery of oncogene inhibitor}

The same adenoviral dodecahedron was used to deliver oncogene inhibitor to the rat orthotopic model of HCC. Initiation factor eIF4E is an oncogene that shows elevated expression in human cancers, which is linked with poor prognosis. For this reason a cap structure analog (cap) that is an eIF4E inhibitor, was conjugated to Dd. This small oncogene inhibitor is cell impermeant when free. The application of Dd-cap together with Dd-dox (anti-cancer antibiotic doxorubicin) resulted not only in inhibition of cancer cell culture proliferation in vitro, but in addition, significantly inhibited HCC tumour growth in the animal HCC model. Interestingly, two oncogenes, eIF4E and c-myc known to be responsible for tumour maintenance and progression, were extinguished in tumour sections of treated rats (Zochowska et al., 2015) (Fig. 2).

Delivery of nucleic acids. VLPs in gene therapy

VLPs can also be employed to transfer nucleic acids, thanks to encapsulation. For example, when bacteriophage MS2 VLPs containing miRNA were prepared, miRNA was inserted into a prokaryotic expression vector and co-expressed with the capsid protein of bacteriophage MS2 using the prokaryotic expression system (Pan et al., 2012). After purification, VLPs containing miRNA were administered to lupus erythematosus-prone mice. Treatment with MS2-miRNA VLP resulted in 
a significant reduction in the expression of autoantibodies (sign of lupus). Remarkably, these mice also exhibited reduced levels of pro-inflammatory cytokines (op. cit.).

\section{CONCLUSION}

Current research shows increasing popularity of VLPs, since these versatile, multimeric nanostructures can have many applications in vaccinology, gene therapy or drug delivery. The VLPs features important for drug delivery are cell-specific targeting, efficient cell entry, lack of endosomal sequestering, multivalency and biocompatibility. A large variety of active molecules can be encapsulated into the VLPs or attached to it. Since VLPs are devoid of genetic material, they provide safe delivery systems without any risk of infection.

The use of VLP as a vaccination platform is already a medical evidence, however, the studies on VLPs as drug delivery vectors are in their infancy. The greatest hurdle in their utilization for drug delivery is the immune response against these proteinaceous vectors, which might relegate VLPs use to auxiliary therapeutic applications, for example upon the failure of classical chemotherapy.

Figure captions

\section{Acknowledgements}

This work was financially supported by the National Science Centre (Poland) based on the decision number DEC-2013/09/B/NZ3/02327.

\section{REFERENCES}

Ashley CE, Carnes EC, Phillips GK, Durfee PN, Buley MD, Lino CA, Padilla DP, Phillips B, Carter MB, Willman CL, Brinker CJ, Caldeira Jdo C, Chackerian B, Wharton W, Peabody DS (2011) Cell-specific delivery of diverse cargos by bacteriophage MS2 viruslike particles. ACS Nano 5: 5729-5745. doi: 10.1021/nn201397z.

Bareford LM, Swaan PW (2007) Endocytic mechanisms for targeted drug delivery. Adv Drug Deliv Rev 59: 748-758.

Chroboczek J, Szurgot I, Szolajska E (2014) Virus-like particles as vaccine. Acta Biochim Pol 61: 531-539.

Cortes-Perez NG, Sapin C, Jaffrelo L, Daou S, Grill JP, Langella P, Seksik P, Beaugerie L, Chwetzoff S, Trugnan G (2010) Rotaviruslike particles: a novel nanocarrier for the gut. J Biomed Biotechnol 2010: 317545. doi: 10.1155/2010/317545.

Crisci E, Bárcena J, Montoya M (2012) Virus-like particles: The new frontier of vaccines for animal viral infections. Vet Immunol Immunopathol 148: 211-225. doi: 10.1016/j.vetimm.

Douglas T, Young M (1998) Host-guest encapsulation of material by assembled virus protein cages. Nature 393: 152-155.

Estes M, Kapikian AZ (2007) Rotaviruses. In Fields virology, Knipe DM, Howley PM, Griffin DE, Lamb RA, Martin MA, Roizman B, Straus SE eds 5th edn, pp 1917-1974. Lippincott Williams \& Wilkins, Philadelphia, PA.

Fender P, Ruigrok RW, Gout E, Buffet S, Chroboczek J (1997) Adenovirus dodecahedron, a new vector for human gene transfer. Nat Biotechnol 15: 52-56.

Frietze KM, Peabody DS, Chackerian B (2016) Engineering viruslike particles as vaccine platforms. Curr Opin Virol 18: 44-49. doi: 10.1016/j.coviro.2016.03.001.

Grasso S, Santi L (2010) Viral nanoparticles as macromolecular devices for new therapeutic and pharmaceutical approaches. Int $J$ Physiol Pathophysiol Pharmacol 2: 161-178.

Guu TS, Liu Z, Ye Q, Mata DA, Li K, Yin C, Zhang J, Tao YJ (2009) Structure of the hepatitis $\mathrm{E}$ virus-like particle suggests mechanisms for virus assembly and receptor binding. Proc Natl Acad Sci USA 106: 12992-12997. doi: 10.1073/pnas.0904848106.
Ishizu KI, Watanabe H, Han SI, Kanesashi SN, Hoque M, Yajima H, Kataoka K, Handa H (2001) Roles of disulfide linkage and calcium ion-mediated interactions in assembly and disassembly of virus-like particles composed of simian virus 40. VP1 capsid protein. J Virol 75: 61-72.

Jian F et al. (2012) Toxicity of biodegradable nanoscale preparations. Curr Drug Metab 13: 440-446.

Kushnir N, Streatfield SJ, Yusibov V (2012) Virus-like particles as a highly efficient vaccine platform: Diversity of targets and production systems and advances in clinical development. Vaccine 31: 5883. doi: 10.1016/j.vaccine.

Lua LH, Connors NK, Sainsbury F, Chuan YP, Wibowo N, Middelberg AP (2014) Bioengineering virus-like particles as vaccines. Biotechnol Bioeng 111: 425-440. doi: 10.1002/bit.25159.

Ma Y, Nolte RJ, Cornelissen JJ (2012) Virus-based nanocarriers for drug delivery. Adv Drug Deliv Rev 64: 811-825. doi: 10.1016/j. addr.2012.01.005.

Pan Y, Jia T, Zhang Y, Zhang K, Zhang R, Li J, Wang L (2012) MS2 VLP-based delivery of microRNA-146a inhibits autoantibody production in lupus-prone mice. Int J Nanomedicine 7: 5957-5967. doi: 10.2147/IJN.S37990.

Park JH, Choi EA, Cho EW, Lee YJ, Park JM, Na SY, Kim KL (2000) Detection of cellular receptors specific for the hepatitis $B$ virus preS surface protein on cell lines of extrahepatic origin. Biochem Biophys Res Commun 277: 246-254.

Peretti S, Schiavoni I, Pugliese K, Federico M (2006) Selective elimination of HIV-1-infected cells by Env-directed,HIV-1-based virus-like particles Virology 345: 115-126.

Perrone LA, Ahmad A, Veguilla V, Lu X, Smith G, Katz JM, Pushko P, Tumpey TM (2009) Intranasal vaccination with 1918 influenza virus-like particles protects mice and ferrets from lethal 1918 and H5N1 influenza virus challenge. J Virol 83: 5726-5734. doi: 10.1128/JVI.00207-09.

Rynda-Apple A, Patterson DP, Douglas T (2014) Virus-like particles as antigenic nanomaterials for inducing protective immune responses in the lung. Nanomedicine (Lond) 9: 1857-1868. doi: 10.2217/ nm.14.107.

Sebestik J et al. (2011) Peptide and glycopeptide dendrimers and analogous dendrimeric structures and their biomedical applications. Amino Acids 40: 301-370. doi: 10.1007/s00726-010-0707-z.

Shin YC, Folk WR (2003) Formation of polyomavirus-like particles with different VP1 molecules that bind the urokinase plasminogen activator receptor. I Virol 77: 11491-1148.

Shete HK, Prabhu RH, Patravale VB (2014) Endosomal escape: a bottleneck in intracellular delivery. I Nanosci Nanotechnol 14: 460-474.

Shlomai A, Lubelsky Y, Har-Noy O, Shaul Y (2009) The "Trojan horse" model-delivery of anti-HBV small interfering RNAs by a recombinant HBV vector. Biochem Biophys Res Commun 390: 619-623. doi: 10.1016/j.bbrc.2009.10.016.

Smith GE, Flyer DC, Raghunandan R, Liu Y, Wei Z, Wu Y, Kpamegan E, Courbron D, Fries LF 3rd, Glenn GM (2013) Development of influenza H7N9 virus like particle (VLP) vaccine: homologous A/Anhui/1/2013 (H7N9) protection and heterologous A/chicken/ Jalisco/CPA1/2012 (H7N3) cross-protection in vaccinated mice challenged with H7N9 virus. Vaccine 31: 4305-4313. doi: 10.1016/j. vaccine.2013.07.043.

Spohn G, Jennings GT, Martina BE, Keller I, Beck M, Pumpens P, Osterhaus AD, Bachmann MF (2010) A VLP-based vaccine targeting domain III of the West Nile virus E protein protects from lethal infection in mice. Virol J 7: 146. doi: 10.1186/1743-422X-7-146.

Van den Berg A, Dowdy SF (2013) Protein transduction domain delivery of therapeutic macromolecules. Curr Opin Biotechnol 22: 888-893. doi: 1016/j.copbio.2011.03.008.

Zeltins A (2013) Construction and characterization of virus-like particles: a review. Mol Biotechnol 53: 92-107. doi: 10.1007/s12033-0129598-4.

Zochowska M, Piguet AC, Jemielity J, Kowalska J, Szolajska E, Dufour JF, Chroboczek J (2015) Virus-like particle-mediated intracellular delivery of mRNA cap analog with in vivo activity against hepatocellular carcinomas. Nanomedicine 11: 67-76. doi: 10.1016/j. nano.2014.07.009.

Zochowska M, Paca A, Schoehn G, Andrieu JP, Chroboczek J, Dublet B, Szolajska E (2009) Adenovirus dodecahedron, as a drug delivery vector. PLoS One 4: e5569. doi: 10.1371/journal.pone.0005569. 